\title{
Analytical Description of SMA Actuator Dynamics based on Fermi-Dirac Function
}

\author{
K. KLuszCZyŃSKI AND M. KCIUK* \\ Silesian University of Technology, Faculty of Electrical Engineering, Chair of Mechatronics, \\ Akademicka 10a, 44-100 Gliwice, Poland
}

\begin{abstract}
The paper deals with possible application of the very promising SMART material - shape memory alloy. At the beginning the laboratory stand for investigation on dynamic behaviour of a SMA linear actuator is presented and results of measurements for the chosen SMA actuator F2000 are depicted and discussed. In the next stage the authors propose to use the so-called Fermi-Dirac function for description of SMA linear actuator dynamics. Applying this function for determining basic performance curve: shortening $\Delta L$ versus time $t$ for different loads $F$ requires proper modification of the Fermi-Dirac function and its conversion in the one-parameter family (with parameter $F$ ). Coefficients of this family are determined with the help of Hooke-Jeeves optimization algorithm and on the basis of experimental findings separately for activation and deactivation process. The both derived expressions were validated by additional laboratory investigations. Finally, the elaborated descriptions was employed in design procedure of a robot gripper. It was shown that the results of measurements for the gripper prototype are in satisfactory agreement with the results of calculations.
\end{abstract}

DOI: 10.12693/APhysPolA.131.1274

PACS/topics: 87.85.St, 71.20.Gj, 71.20.Be

\section{Introduction - SMA materials - definition, properties and possible applications}

Shape memory alloy (SMA) belongs to materials known as SMART materials (it means that one of their features can be controlled by external signal). In case of SMA material it is ability for keeping memory about its primary shape and the essence of this phenomenon is related to its two possible phases (material structure): martensitic phase (connected with low temperature scope and characterized by a disordered structure) and austenitic phase (related to high temperature scope and associated with a crystalline structure). In martensitic phase it is quite easy to change the material shape but under high temperature (it means - in austenitic phase) the material becomes stiff and turns back to its primary (initial) shape [1]. Control signal starting the shape change is temperature rise (e.g. flame [2], infrared rays etc.) or flow of current (Joule's heat) leading to temperature increase (in the second case we speak about a current-controlled SMA material). The process of changing material structure under varying thermal condition is called martensitic-austenitic transition ( $\mathrm{m}-\mathrm{a}$ transition) and can be described graphically by nonlinear curve: ratio $\xi$ vs. temperature, where ratio $\xi$ expresses relative martensitic phase content in the whole considered material (Fig. 1).

Transition starts at the initial temperature $T_{i}^{m-a}(\xi=$ 1) and finishes at the final temperature $T_{f}^{m-a}(\xi=0)$ (see "m-a branch" in Fig. 1). This process is reversible but it is necessary to notice that inverse $\mathrm{a}-\mathrm{m}$ transition starts

*corresponding author; e-mail:

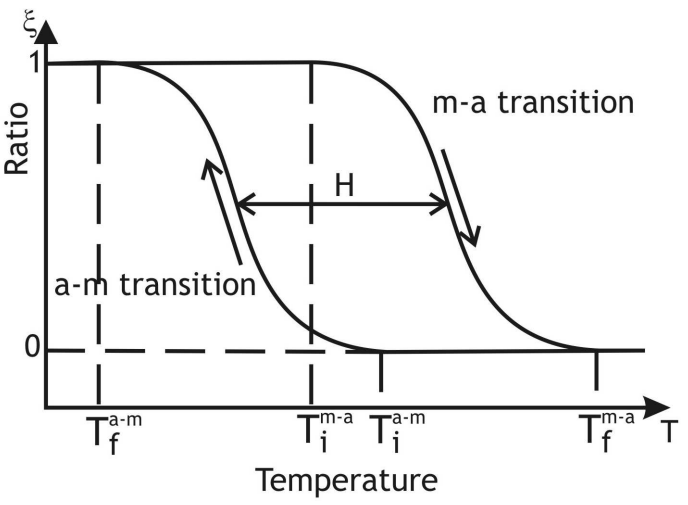

Fig. 1. Martensitic and austenitic transition of SMA.

at the another temperature $T_{i}^{a-m}$ and finishes at the another temperature $T_{f}^{a-m}$, as well (see "a-m branch" in Fig. 1). It means that full cycle (direct and inverse transition) is described by a double-valued curve (hysteresis loop).

It is worth to underline that the values of the abovedefined four critical temperatures depend considerably on intensity of mechanical stress caused by external force $F$, which is shown in Fig. 2.

It results from Fig. 2 the higher external force $F$ (stress), the higher values of critical temperatures: $T_{i}^{m-a}, T_{f}^{m-a}, T_{i}^{a-m}, T_{f}^{a-m}[3]$.

There are many research and educational projects oriented at different possible application of SMA actuators in electromechanical and mechatronic systems. It results from the fact that SMA actuators offer many unique advantages among whose the most important is very simple and very light mechanical construction [4-8].

SMA are also used as active (controlled) or passive (not controlled) actuators. Passive actuators exploit the phe- 


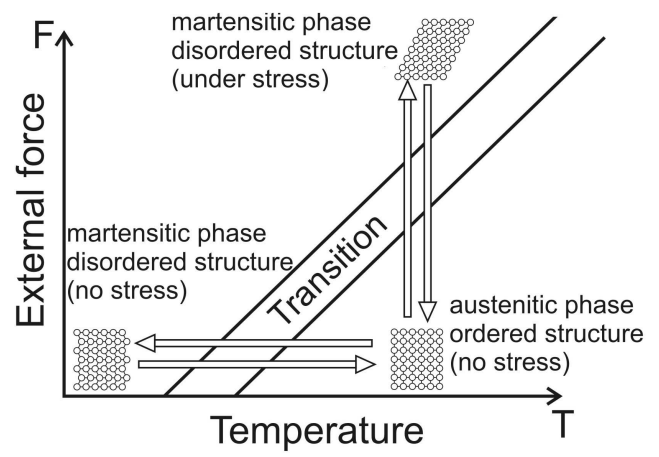

Fig. 2. External force influence on transition temperatures.

nomenon of superelasticity. Some examples of their practical applications are presented in literature. They are used in civil engineering to protect buildings or bridges exposed to earthquakes (e.g. as seismic protection system) [9]. Passive SMA actuators can suppress vibrations. Example of a SMA/rubber isolation system is presented in Fig. 3.

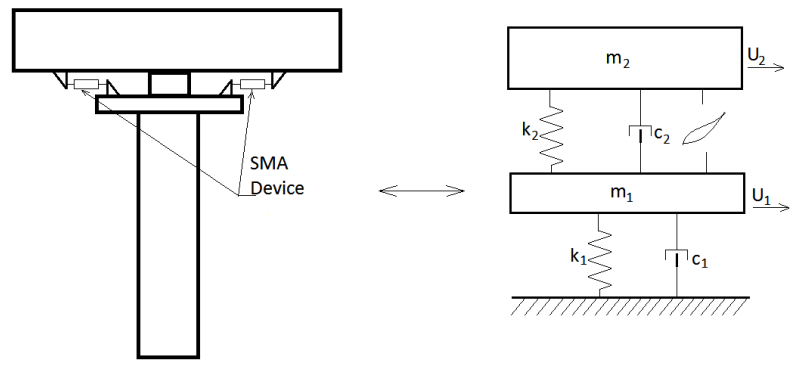

Fig. 3. Bridge with SMA/rubber isolation system: (a) model of an isolated bridge, (b) equivalent circuit of mechanical construction.

Active SMA actuators are popular in mechatronic applications as unconventional drives. They belong to the group of light-weight actuators with high value of power to weight ratio and can be recommended as reliable and cheap drive system performing simple tasks (e.g. displacement, rotation, pooling, lifting etc.). What is more, they ensure sparkless and safety work in flammable areas. It is also possible to build light-weight robots (or robotic tools) actuated by SMAs. The unconventional robotic hand driven by SMAs is described in [6]. Two another types of unconventional mobile robots (snake and net robots) are presented in $[5,10]$. It is also possible to build micro-robots made with MEMS technology, actuated by SMAs [4].

\section{Basic information of SMA linear actuators}

An SMA actuator is an electromechanical converter in which electric energy is converted into mechanical energy via thermal energy [11]. The actuator (in form of a rod, a spring or a piston) made of shape memory alloy may be biased by another SMA actuator, spring or gravitational load (Fig. 4). For each case depicted in Fig. 4 one can recognize another characteristics: force $F$ vs. displacement. This characteristics are put together in the bottom of Fig. 4.

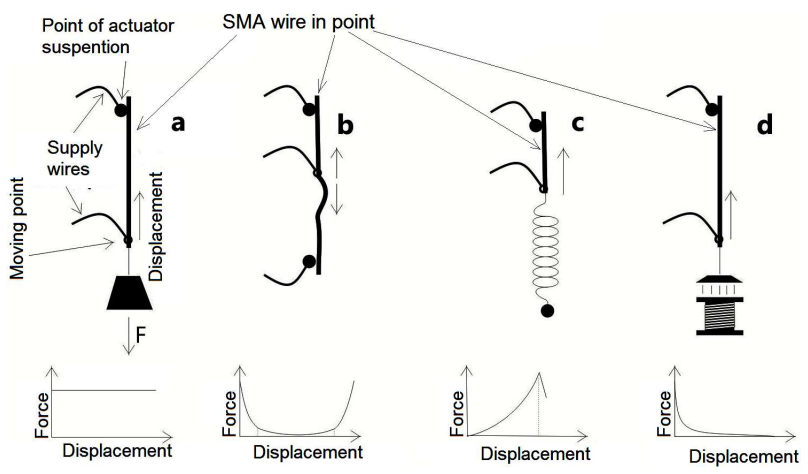

Fig. 4. Examples of different SMA actuator biases: a) - gravity, b) — oposing wires, c) — spring, d) electromagnet.

Change of temperature is exerted by a current stimulus and leads to a linear actuator deformation. In the low temperature state (martensitic phase) referred to the deactivation state (state with no current stimulation), the material follows the loading force, thus an elongation of the actuator is observed. The austenitic phase (the activation state) is stimulated by the increase of temperature above the threshold value (minimum current flow) and in spite of the load causes turn of the alloy to the "memorized" initial state (an action against the loading force expressing in the shortening of an actuator $\Delta L)$. High temperature state (austenite phase) is termed activation state because the actuator keeps a "memorized" position acting against the mechanical force.

Cooling process is called deactivation process because internal stresses in the material are fading out gradually and, as a result, actuator is deformed.

\section{Laboratory stand for investigation on SMA actuators}

The laboratory stand for investigation of an individual linear actuator having an arbitrary form (a rod, a piston or a spring) is presented in Fig. 5. An actuator is fed from the power supply PSH-3620A (36 V, $20 \mathrm{~A})$ which enables us to keep constant value of stimulating current in spite of varying value of actuator resistance.

Stimulating current is considered as control variable which allows to set the given length of the shortened actuator. Shortening of the actuator $\Delta L$ is measured by the optical displacement sensor Q9871. Temperature measurement is realized using the infrared camera (IR camera) Flir A325 which is commonly used in wide range of SMART material investigations e.g. [13]. It has resolution $320 \times 240$ pixels and can record pictures up to $60 \mathrm{~Hz}$. In order to improve quality of infrared measurement, final part of the camera with lenses and the investigated actuator are covered with a black cardboard sleeve. Temperature of the actuator depends mainly on value of stimulating current (according to the Joule-Lenz law temperature 
rise of an actuator is proportional to the square of stimulating current) but is also influenced by ambient temperature which is continuously measured by the digital thermometer DS18B20. Essential advantage of the laboratory stand in point is that the investigated actuator can be easily manually loaded in gravitational way by the weights of growing values up to $2 \mathrm{kG}$. As seen in Fig. 6a, the laboratory stand is additionally equipped with the box of the four precise multimeters Rigol DM3052, as well as the digital oscilloscope Tektronix MSO 2024 and PC computer responsible for controlling signals, data acquisition and data processing (which are not seen in Fig. 6a).

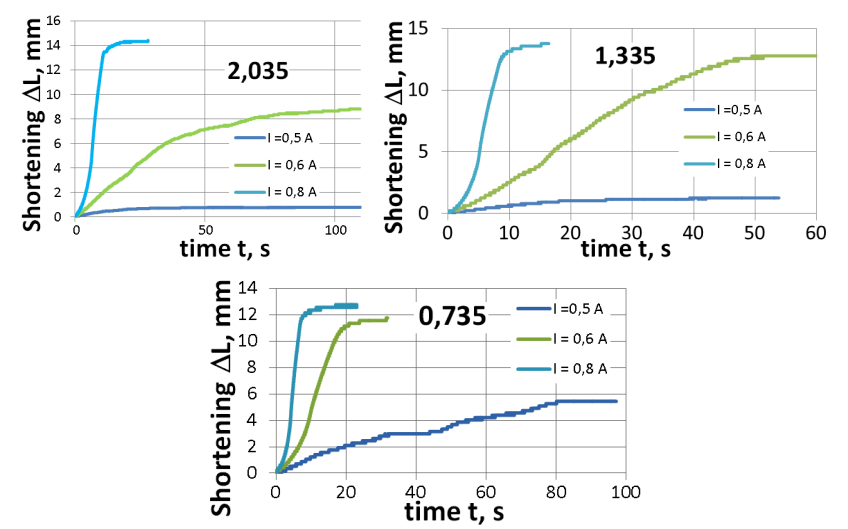

Fig. 5. Basic time curves: shortening $\Delta L$ vs. time $t$ for different values of excitation current $(I=0.5,0.6,0.8 \mathrm{~A})$ and at different loads $(F=2.035,1.335,0.735 \mathrm{kG})$.

a)

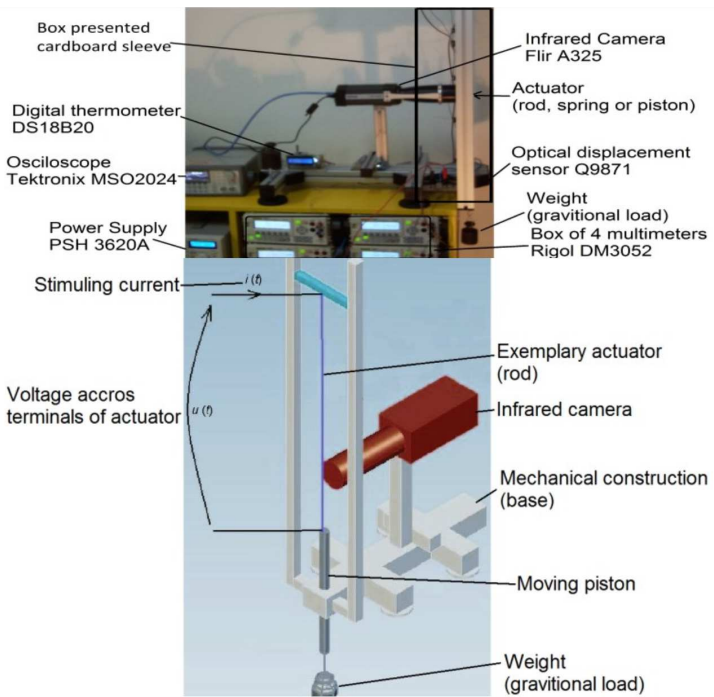

Fig. 6. Laboratory stand for investigation on individual SMA linear actuator: (a) general view (photo), (b) computer visualization.

\section{Investigation on dynamic behaviour of SMA linear actuator}

The elaborated laboratory stand described in the foregoing chapter and depicted in Fig. 6 enables us to perform measurements oriented at dynamic properties of
SMA linear actuator. As representative item the authors choose linear actuator F2000 manufactured by DYNALLOY and having the following rated data: nominal force $F_{N}=2 \mathrm{kG}$, nominal excitation current $I_{N}=0.8 \mathrm{~A}$, nominal length $L_{N}=384 \mathrm{~mm}$ and minimum length $L_{0}=263 \mathrm{~mm}$.

During investigation the SMA linear actuator was many times activated and deactivated under different conditions and both: activation process (related to heating action) and deactivation process (connected with natural cooling phenomenon) were observed and measured in order to determinate basic time curves: shortening $\Delta L$ vs. time $t$. The results of investigation carried out for the different values of excitation currents $(I=0.5,0.6$, $0.8 \mathrm{~A})$ and at the various values of gravitational loads $(F=0.735,1.335,2.035 \mathrm{kG})$ are presented in Fig. 5 .

From viewpoint of nominal data the SMA actuator was activated by the relative excitation currents equal to: $62.5 \%, 75 \%$ and $100 \%$ and loaded by the relative force equal to: $37 \%, 67 \%$ and $1.02 \%$. In all cases the load includes besides metric system weights also weight of the moving piston (Fig. 7).

The obtained results confirm that the rated data delivered by the manufacturer are correct and that the excitation current $I=0.8 \mathrm{~A}$ and the load $F=2 \mathrm{kG}$ can be treated further as nominal data of the SMA linear actuator F2000 (certified by the authors on their own on the elaborated laboratory stand).

In other words the investigated SMA linear actuator can be used in any drive system as a two-state actuator excited by the nominal current $I_{N}=0.8 \mathrm{~A}$ and offering displacement of the given mass (not greater than $2 \mathrm{~kg}$ ) for distance about $12-14 \mathrm{~mm}$ which is equal to about $3.5 \%$ of the actuator length.

Of course, instead of displacement one can speak about the force $2 \mathrm{kG}$ acting upon the given object or pulling spring (Fig. 4). Dynamic performance curves of the considered two-state actuator for activation process (heating) and deactivation process (cooling) are put together in Fig. 8, respectively.

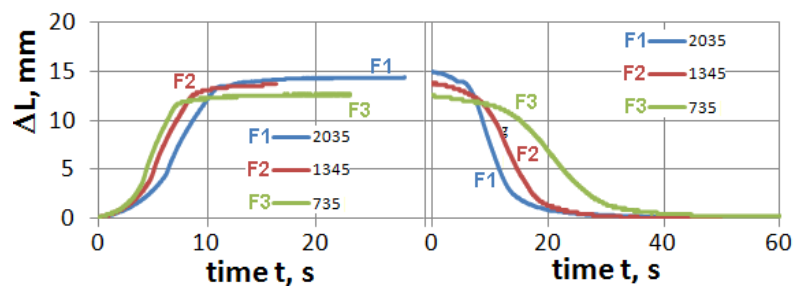

Fig. 7. Dynamic performance curves of two-state actuators at different loads and for nominal excitation current $\left(I_{N}=0.8 \mathrm{~A}\right)$ expressed graphically in form of oneparameter family: (a) activation, (b) deactivation.

Looking at these curves one can easily perceive that large number of the former-mentioned advantages of the SMA actuator (simple construction, high value of power per weight ratio etc.) have been overshadowed by very poor dynamic properties: long time of activation related 
to thermal phenomenon and about twice longer time of deactivation resulting from slowness of self-cooling process.

It causes that application of SMA actuators are limited only to the group of drive systems which do not require high dynamics and quick reaction to control signal.

For purpose of such slowly-acting mechatronic systems there is need to elaborate analytical description of SMA actuator dynamics which allows to implement SMA drives in mechatronic systems.

\section{Analytical description of SMA actuator dynamics employing the modified Fermi-Dirac function}

Considering the slopes of the consecutive actuation curves presented in Fig. 7 one can easily notice four characteristic features which depend on level of load. These are: steady-state (maximum) value of the curve, slope of the function in the middle part of linear region, location of inflection point and slope of the function at the point $t=0$.

After theoretical considerations and on the basis of the engineering intuition the authors decided to choose for further detailed analysis the so-called Fermi-Dirac function (which in some previous papers was applied effectively for the description of steady-state hysteresis curve of SMA linear actuators $[11,12])$, as well as to find analytical description of SMA actuator dynamics in the form of one-parameter family of functions: $\Delta L=f(t, F)$. Such a family of curves are related to common equation so that all curves can be generated by varying parameter $F$ in the equation.

The Fermi-Dirac function (firstly used in physics for describing probability of electron distribution) has the following standard form:

$$
f_{F D}(t)=\frac{K}{1+\exp }(p(c-t)),
$$

where $t$ - independent variable, $K, p, c$ - function coefficients

What is important and very useful all these coefficients $K, p, c$ have very clear mathematical interpretation and can be easily connected with important features of the function. As seen in the consecutive plots in Fig. 8, coefficient $K$ is equal to the right-hand limit of the FermiDirac function: $f_{F D}(t) \rightarrow K$ (see Fig. $8 \mathrm{a}$ ), $c$ is equal to value of time $t$ at which the Fermi-Dirac function reaches value $\frac{K}{2}$ (see Fig. 8b) and $p$ determines slope of the function at the point $t=c: p=\left.\frac{\mathrm{d} f_{F D}}{\mathrm{~d} t}\right|_{t=c}$ (see Fig. 8c).

Continuing investigation of the function, it can be proved in simple way that $t=c$ indicates location of inflection point: $\left.\frac{\mathrm{d}^{2} f_{F D}}{\mathrm{~d} t^{2}}\right|_{t=c}=0$ and that slope of function at the point $t=0$ can be calculated according to the following expression:

$$
\left.\frac{\mathrm{d} f_{F D}}{\mathrm{~d} t}\right|_{t=0}=\frac{K P \exp (p c)}{(\exp (p c)+1)^{2}}
$$

Bearing in mind the above conclusions the authors

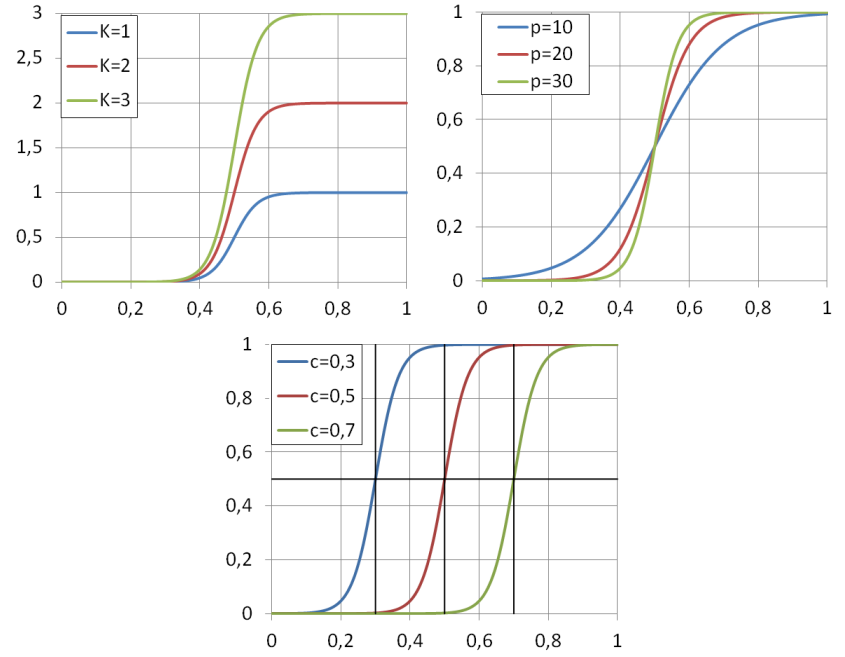

Fig. 8. Influence of parameters $K, p, c$ on Fermi-Dirac functions.

decided to modify standard Fermi-Dirac function (Eq. (5.1)) and to adopt it for the description of SMA actuator dynamics by converting its standard form in the one-parameter family

$$
\Delta L=f_{F D}(t, F)=\frac{K}{1+\exp (p(c-t))}
$$

As seen, all constant coefficients of the Fermi-Dirac function were transformed in functions of independent variable $F$ which plays role of parameter.

Because the dynamic curves for activation differ from dynamic curves for deactivation it is necessary to create two one-parameter families - one for activation process

$$
\Delta L^{\uparrow}=\frac{K}{1+\exp \left(p^{\uparrow}\left(c^{\uparrow}-t\right)\right)},
$$

and the second one for deactivation process

$$
\Delta L^{\downarrow}=\frac{K}{1+\exp \left(p^{\downarrow}\left(t-c^{\downarrow}\right)\right)}
$$

It was time-consuming task to find the most suitable analytical forms of functions: $K, p^{\uparrow}, p^{\downarrow}, c^{\uparrow}$ and $c^{\downarrow}$ included in Eqs. (5.4) and (5.5) (in graphical form they are presented in Fig. 9).
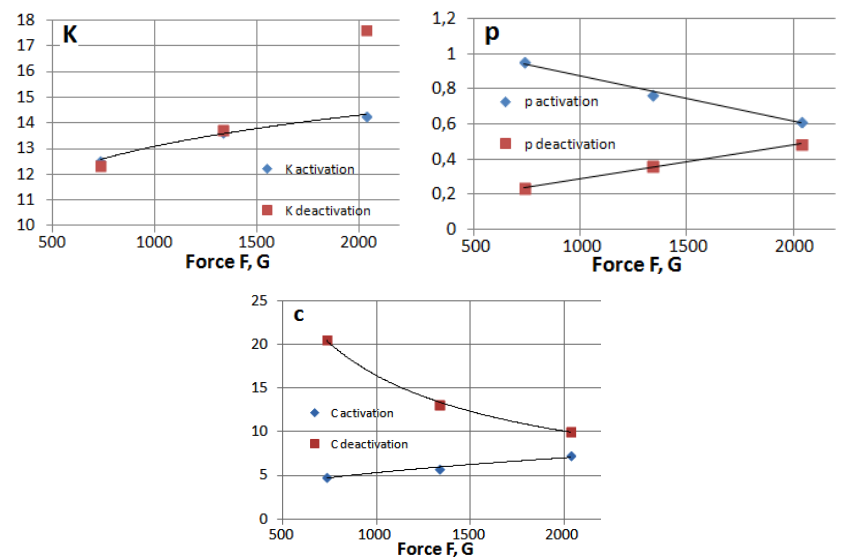

Fig. 9. Coefficients of Fermi-Dirac functions for the applied load. 
After many trials the authors selected as the most proper form of the exponential functions and, as a consequence, the one-parameter families: "shortening $\Delta L$ vs. time $t$ for both activation and deactivation process" receive, finally, the following forms:

$$
\begin{aligned}
\Delta L^{\uparrow} & =\frac{K_{0} F^{\alpha}}{1+\exp \left(\left(\beta_{\uparrow} F+p_{0 \uparrow}\right)\left(c_{0 \uparrow} F^{\gamma \uparrow}-t\right)\right)}, \\
\Delta L^{\downarrow} & =\frac{K_{0} F^{\alpha}}{1+\exp \left(\left(\beta_{\downarrow} F+p_{0 \downarrow}\right)\left(c_{0 \downarrow} F^{\gamma \downarrow}-t\right)\right)} .
\end{aligned}
$$

The values of the factors in Eqs. (5.6): $K_{0}, \quad \alpha, \quad \beta^{\uparrow}, \quad \beta^{\downarrow}, p_{0}^{\uparrow}, \quad p_{0}^{\downarrow}, \quad c_{0}^{\uparrow}, \quad c_{0}^{\downarrow}, \quad \gamma^{\uparrow}, \quad \gamma^{\downarrow}$ calculated on the basis of the measured curves in Fig. 7 and with the help of the Hooke--Jeeves optimization algorithm (oriented at the minimum square error between the measured curves and the modelled curves) for the SMA linear actuator F2000 in point are put together in Table I.

\section{TABLE I}

The values of Eq. (5.6) factors.

\begin{tabular}{c|c|c}
\hline \hline & Activation $(\uparrow)$ & Deactivation $(\downarrow)$ \\
\hline$K_{0}$ & \multicolumn{2}{|c}{4.461} \\
\cline { 2 - 3 }$\alpha$ & \multicolumn{2}{|c}{0.1539} \\
\cline { 2 - 3 }$\beta$ & -0.0003 & 0.0002 \\
$p_{0}$ & 1.16 & 0.0643 \\
$c_{0}$ & 0.4267 & 2895.3 \\
$\gamma$ & 0.3665 & -0.745
\end{tabular}

For the loads $F=0.735,1.335$ and $2.035 \mathrm{kG}$ their values are listed in Table II.

TABLE II

Direct factors for given loads.

\begin{tabular}{c|c|c|c||c|c|c}
\hline \hline & \multicolumn{3}{|c||}{ Activation $(\uparrow)$} & \multicolumn{3}{c}{ Deactivation $(\downarrow)$} \\
\hline$F$ & $K$ & $p$ & $c$ & $K$ & $p$ & $c$ \\
\hline 0.735 & 12.723 & 1.116 & 3.835 & 12.723 & 0.1495 & 23.011 \\
1.335 & 13.724 & 0.955 & 4.697 & 13.723 & 0.2673 & 18.194 \\
2.035 & 14.426 & 0.781 & 5.629 & 14.426 & 0.3946 & 12.988
\end{tabular}

The comparison of the measurement curve and the curve obtained on the basis of analytical formulae at the nominal load are depicted in Fig. 10.

\section{Validation of the Fermi-Dirac analytical description for the arbitrary chosen loads}

In order to confirm correctness and usefulness of the derived analytical forms of functions describing dynamics of SMA actuator (Eq. (5.6)) the additional investigations are performed for the values of loads which were not taken into account during determination of the considered coefficients $K_{0}, \alpha, \beta^{\uparrow}, \beta^{\downarrow}, p_{0}^{\uparrow}, p_{0}^{\downarrow}, c_{0}^{\uparrow}, c_{0}^{\downarrow}, \gamma^{\uparrow}, \gamma^{\downarrow}$. The measured and calculated time curves (shortening, control signal and load) for the selected case: $F=$ $0.545 \mathrm{kG}$ are presented in Fig. 11.

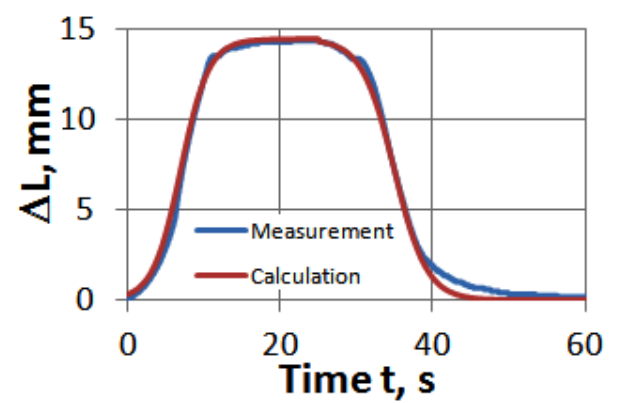

Fig. 10. Comparison of measured and calculated dynamic curves at the nominal load during activation and deactivation process (nominal excitation current $\left.I_{N}=0.8 \mathrm{~A}\right)$.

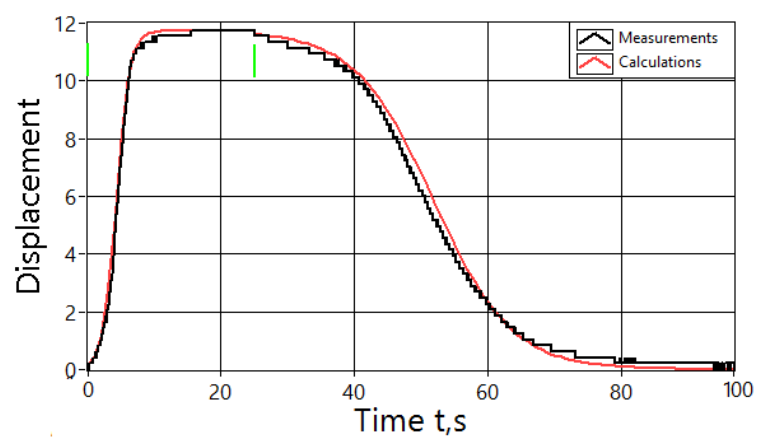

Fig. 11. Comparison of measured and calculated displacement at load $F=0.545 \mathrm{kG}$ (which was not taken into account during determination of coefficients related to one-parameter family of functions). Vertical bars current on and off moments.

The results of measurements, as well as the results of calculations are in satisfactory agreement. It means that the analytical description of SMA actuator dynamics can be used for any value of the force $F$.

\section{The application of analytical description of SMA actuator dynamics in design procedure}

The analytical description of dynamics was used in computer algorithm for designing robot gripper and allowed to calculate properly time of gripping and releasing.

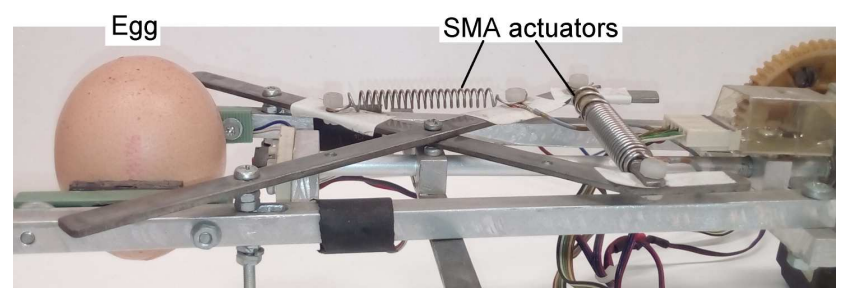

Fig. 12. Robot gripper with egg (example of fragile object).

The time of gripping was equal to $5 \mathrm{~s}$ and the time of releasing was equal to $22 \mathrm{~s}$ (about 4.4 times longer than time of gripping). 
Calculations and experiments made evident that mechatronic systems with SMA actuator have poor dynamics and that time of releasing can be several times longer than time of gripping. In spite of poor dynamic properties SMA grippers can be recommended as grippers for soft and fragile objects (see the grippers with egg in Fig. 12) and in authors' opinion can find application in medicine or biology.

\section{References}

[1] A. Ziółkowski, Habilitation Thesis, IPPT PAN Warszawa 2006 (in Polish).

[2] D. Congalton, Fire Mater. 23, 223 (1999).

[3] B. Selden, K.-J. Cho, H.H. Assada, in: Int. Conf. Robotics Automation, ICRA '04 IEEE International Conference, 2004, p. 4931.

[4] K. Ikuta, in: IEEE International Conference on Robotics and Automation, Vol. 3, Cincinnati $(\mathrm{OH})$ 1990 , p. 2156.

[5] C.Y. Liu, W. H. Liao, in: 2004 IEEE International Conference on Robotics and Biomimetics, Shenyang 2004, p. 601.
[6] K.-J. Cho, H.H. Assada, in: Proc. 2005 IEEE International Conference Robotics Automation, 2005, p. 1356.

[7] M. Bodnicki, D. Kamiński, Mechatronics 2013, Recent Technological and Scientific Advances, Springer, 2014.

[8] M. Kurzawa, D. Stachowiak, Arch. Electr. Eng., to be published.

[9] O.E. Ozbulut, S. Hurlebaus, Smart Mater. Struct. 20, 015003 (2010)

[10] M. Fujii, H. Yokoi, in: Proc. 2003 IEEE Int. Symp. on Computational Intelligence in Robotics and Automation, Vol. 1, Kobe 2003, p. 253.

[11] K. Kluszczyński, M. Kciuk, COMPEL 32, 1417 (2013).

[12] M. Kciuk, K. Chwastek, K. Kluszczyński, J. Szczygłowski, Sens. Actuat. A Phys. 243 , 52 (2016).

[13] P. Kowol, Z. Pilch, Electr. Rev. 91, (2015). 